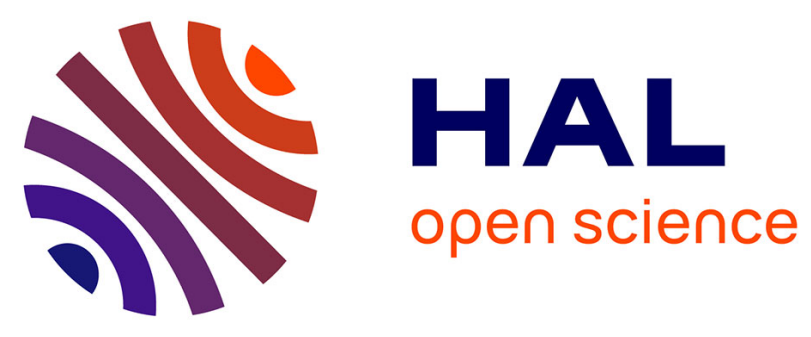

\title{
Distributed Multiuser MIMO for LiFi in Industrial Wireless Applications
}

Kai Lennert Bober, Sreelal Maravanchery Mana, Malte Hinrichs, Sepideh

Mohammadi Kouhini, Christoph Kottke, Dominic Schulz, Christian Schmidt, Ronald Freund, Volker Jungnickel

\section{To cite this version:}

Kai Lennert Bober, Sreelal Maravanchery Mana, Malte Hinrichs, Sepideh Mohammadi Kouhini, Christoph Kottke, et al.. Distributed Multiuser MIMO for LiFi in Industrial Wireless Applications. Journal of Lightwave Technology, 2021, 39 (11), pp.3420-3433. 10.1109/JLT.2021.3069186 . hal03341353

\section{HAL Id: hal-03341353 \\ https://hal-amu.archives-ouvertes.fr/hal-03341353}

Submitted on 10 Sep 2021

HAL is a multi-disciplinary open access archive for the deposit and dissemination of scientific research documents, whether they are published or not. The documents may come from teaching and research institutions in France or abroad, or from public or private research centers.
L'archive ouverte pluridisciplinaire HAL, est destinée au dépôt et à la diffusion de documents scientifiques de niveau recherche, publiés ou non, émanant des établissements d'enseignement et de recherche français ou étrangers, des laboratoires publics ou privés.

\section{(c)(1)}

Distributed under a Creative Commons Attribution| 4.0 International License 


\title{
Distributed Multiuser MIMO for LiFi in Industrial Wireless Applications
}

\author{
Kai Lennert Bober ${ }^{\circledR}$, Student Member, IEEE, Sreelal Maravanchery Mana ${ }^{\circledR}$, Malte Hinrichs ${ }^{\circledR}$, Member, IEEE, \\ Sepideh Mohammadi Kouhini ${ }^{\circledR}$, Student Member, IEEE, Christoph Kottke ${ }^{\circledR}$, Dominic Schulz ${ }^{\circledR}$, \\ Christian Schmidt ${ }^{\circledR}$, Ronald Freund ${ }^{\circledR}$, and Volker Jungnickel ${ }^{\circledR}$, Member, IEEE
}

(Invited Paper)

\begin{abstract}
We present a concept for networked optical wireless communications, also denoted as $\mathrm{LiFi}$, to meet the requirements of industrial wireless applications. These are primarily mobility support with moderate data rates per device, reliable real-time communication, and integrated positioning. We describe a distributed multiuser multiple-input multiple-output architecture, serving mobile devices via an optical wireless infrastructure. The system consists of a central unit, being connected to a number of distributed optical frontends covering a larger area. Our main contribution is a medium access control protocol based on space division multiple access. Evaluation results demonstrate the advantages of joint transmission from adjacent optical frontends and the dynamic switching between spatial diversity and multiplexing. The relevance of spatial multiplexing becomes obvious through channel measurements in an indoor scenario. Moreover, we highlight a low-power physical layer based on on-off-keying for battery-powered mobile devices. Our architecture can easily integrate positioning by simultaneously measuring the time-of-flight between multiple optical frontends and the mobile device. We highlight the use of plastic optical fiber as an analog fronthaul technology and discuss the integration with other networks. The main functions described in this paper will be supported by the upcoming IEEE Std 802.15.13.
\end{abstract}

Index Terms-Industrial communication, LiFi, MIMO, multiuser, optical propagation, optical wireless communications, spatial diversity.

\section{INTRODUCTION}

$\mathbf{T}$ HE automation of production facilities is steadily advancing and constitutes a core aspect of Industry 4.0 [1] Automation is in large parts enabled by the communication and coordination of production machines. In modern smart factories,

Manuscript received August 2, 2020; revised November 29, 2020 and February 12, 2021; accepted February 22, 2021. Date of publication March 29, 2021; date of current version June 2, 2021. This work was partially supported by the EU in the H2020 projects VisIoN and ELIoT under Grants 764461 and 825651 and in part by the German Federal Ministry of Education and Research in the project SESAM under Contract 16KIS0639K.

The authors are with the Fraunhofer-Institut für Nachrichtentechnik, Heinrich-Hertz-Institut, 10587 Berlin, Germany (e-mail: kai.lennert.bober@ hhi.fraunhofer.de; sreelal.maravanchery@hhi.fraunhofer.de; malte.hinrichs@ hhi-extern.fraunhofer.de; sepideh.kouhini@hhi.fraunhofer.de; christoph kottke@hhi.fraunhofer.de; dominic.schulz@hhi.fraunhofer.de; christian schmidt@hhi.fraunhofer.de; ronald.freund@hhi.fraunhofer.de; volker. jungnickel@hhi.fraunhofer.de).

Color versions of one or more figures in this article are available at https: //doi.org/10.1109/JLT.2021.3069186.

Digital Object Identifier 10.1109/JLT.2021.3069186
TABLE I

Industrial COMmunicAtion PERFormance Classes

\begin{tabular}{cc}
\hline \hline Class & Cycle time order \\
\hline A & $\sim 100 \mathrm{~ms}$ \\
B & $\sim 10 \mathrm{~ms}$ \\
C & $<1 \mathrm{~ms}$ \\
\hline \hline
\end{tabular}

production lines are reconfigured on a regular basis and an increasing number of autonomous robots will transport goods and exchange data with the cloud, where artificial intelligence is available [2]. Wireless communication is key to enabling more flexibility in future production processes.

\section{A. Industrial Wireless Communication}

The field of industrial applications is diverse and a general definition of industrial communication characteristics does not exist. Two high-level groups of applications are factory automation and (motion) control. Typically, factory automation applications have more relaxed requirements on communication than control applications. Cycle times are the primary performance indicators for industrial applications and denote the time in which the application data must perform a round-trip. This may be, for example, the update rate between a sensor and an actuator in a control loop. A common approach is to divide applications and network technologies into three classes A, B, and C. The authors of [3] give an overview over these classes and sketch future directions of industrial communication. Table I lists the three performance classes and corresponding cycle times. Class $\mathrm{C}$ is the most demanding class, including applications like distributed control systems, i.e., control loops between spatially distributed sensors and actuators. Applications of this class require cycle times below $1 \mathrm{~ms}$. Some class $\mathrm{C}$ protocols, such as Profinet, Sercos, or EtherCAT, even support cycle times as low as 32.5 $\mu$ s for small data packets. In class B, longer cycle times up to around $10 \mathrm{~ms}$ are tolerable. Class A applications can handle cycle times in the realm of $100 \mathrm{~ms}$. In order to allow periodic communication within the cycle time, latency and jitter must reside well below the cycle time. For industrial communication, frame loss rates must be very low, ranging in the order of wired technologies. That means that less than one frame in a million 
should be lost or arrive late, with a trend for even lower loss rates for some applications. On the other hand, the throughput is typically moderate, but new applications may challenge this.

Given the increasing need for flexibility and mobility in modern factories, the demand for wireless solutions is intuitive. Technologies tend to replace existing cables and aim to support real-time communication, which imposes high requirements on the robustness and reliability of the wireless link. A range of radio technologies is already used for industrial wireless communication, as summarized in [4]. Technologies based on the IEEE 802.15.4 standard like WirelessHART, ISA100.11a, or ZigBee [5], [6] are popular choices for industrial wireless communication. The use of Bluetooth [7] or DECT [8] is common as well. IEEE Std 802.11, known as Wi-Fi, is also used on factory floors. Lately, cellular network companies have recognized industrial wireless communication as a new vertical market [9]. While 5G mobile networks continue to provide best-effort connectivity to mobile devices, called enhanced mobile broadband (eMBB), 5G can simultaneously operate in further profiles like the ultra-reliable low-latency communications (URLLC) or massive machine-type communication (mMTC). Some countries enable $5 \mathrm{G}$ private networks targeting communication on factory floors [10]. Factory owners can obtain a local $5 \mathrm{G}$ license and deploy a private network on their own property. The authors of [11] compile a number of exemplary use cases and requirements identified for industrial $5 \mathrm{G}$.

Still, radio communication has a natural disadvantage against cables for real-time communication. The wireless medium is susceptible to various disturbances like interference, shadowing, and wideband fading effects which are typical for indoor environments. The authors of [12] show these effects based on extensive measurements of the industrial wireless channel. Consequently, industrial wireless networks are an ongoing research topic with the goal to make wireless communication suitable for more and more demanding applications. The authors of [13] propose a new wireless protocol for control applications, targeting reliable transmission of control type traffic through reserving resources for potential retransmission attempts. The author of [14] discusses the necessity of leveraging spatial diversity over repeated transmission in wireless channels, which arises from the relationship between maximum latencies and the fading or blocking duration of the wireless channel. Apart from delivering the necessary quality of service (QoS), power consumption and security play important roles [4].

Recently, new applications with additional requirements, such as higher throughput, mobility, or support for large number of users, were identified. Examples are edge cloud offloading for augmented reality [15], the networking of mobile robots [16], or the massive deployment of Internet of Things (IoT) devices and sensors for smart manufacturing [17], [18]. The authors of [19] provide an overview over the performance of state-of-the-art industrial wireless technologies and highlight the necessity for new technologies.

\section{B. Light Fidelity (LiFi)}

Optical wireless communications (OWC) has been investigated since the late 1970s [20] under varying names. As an alternative to using radio frequencies for wireless transmission, it exhibits distinctive propagation characteristics such as high directivity, complete containment through walls, and dominant transmission via the line-of-sight (LOS). OWC with multiple networked transceivers, e.g., integrated into luminaires, and mobility support is referred to as $\mathrm{LiFi}$. LiFi transceivers in a cellular layout can reuse the complete communication bandwidth every few meters, resulting in a mobile network with the potential for ultra-high data density. As an example, current Wi-Fi technologies based on IEEE 802.11ac support an average rate of 500 Mbit/s in a typical area of $500 \mathrm{~m}^{2}\left(1 \mathrm{Mbit} / \mathrm{s} / \mathrm{m}^{2}\right)$. The extension $802.11 \mathrm{ax}$ aims at serving significantly more users, achieving $10 \mathrm{Mbit} / \mathrm{s} / \mathrm{m}^{2}$ through advanced interference management. Due to multi-path propagation and interference, however, radio has fundamental limits to increase spectral area efficiency further Emerging LiFi systems are based on LOS propagation and support several hundred Mbit/s within a few $\mathrm{m}^{2}\left(100 \mathrm{Mbit} / \mathrm{s} / \mathrm{m}^{2}\right)$ as reported in [21]. Thus, LiFi networks can complement mobile radio in order to densify the network and add the possibility for traffic offloading. This way, radio frequencies can be reserved for highly mobile users, adding a new dimension to future mobile networks [22].

Various aspects of OWC, such as transceivers, modulation formats, and channel properties, were researched in the past. Recently, modern techniques such as multiple-input multipleoutput (MIMO) [23] and orthogonal frequency division multiplexing (OFDM) modulation format have been used together with $\mathrm{LiFi}$. It was found that direct-current offset OFDM (DCOOFDM) in combination with adaptive bit loading is able to exploit the low-pass channel of OWC efficiently [24]. First devices follow this approach based on commercially available chipsets that are compliant with the International Telecommunication Union Telecommunication Standardization Sector (ITU-T) G.9991 recommendation [25]. Examples are the OLEDCOMM LiFiMAX, Signify Trulifi, and Fraunhofer HHI LiFi Neon systems.

LiFi has particular advantages for industrial wireless communication. The local confinement of light through walls adds a layer of confidentiality on top of encryption and protects factory owners from espionage through competitors. Conversely, LiFi is robust against jamming through (radio) signals transmitted by an adversary outside the building and electromagnetic interference from machines. The narrow light beams allow spatial multiplexing of simultaneous transmissions and therefore reduce queueing delays when many users are active. This makes LiFi suitable to realize high density real-time networks. At the same time, $\mathrm{LiFi}$ networks can be operated independently from any provider or licensing authority, which allows for high planning security and economic efficiency for factory owners.

In [26], energy harvesting and energy efficient modulations are motivated for industrial LiFi. The work in [27] investigates multiple LiFi system designs for IoT, also considering power efficiency. In [28], industrial LiFi channels for different ceiling heights are examined using an optical simulation software. Measurements of the optical channel in [12] confirm that OWC in industrial environments is predominantly impacted by large scale fading and shadowing effects. In [29], the use of MIMO techniques is proposed to make optical communication robust 
against link blockage. The availability of redundant transmission paths introduces resilience and improves the reliability of the optical link.

To assess the LiFi MIMO channel and the fundamental feasibility of the concept in a real industrial environment, we took first broadband measurements with an $8 \times 6$ MIMO-channel sounder in a robotic cell [30]. For the measurements, optical frontends (OFEs) including light-emitting diodes (LEDs) and photodiodes were mounted on the robot's arm and at the cell cage. The results indicated that a sufficient number of LOS links between the robot and the fixed OFEs were available at all points along the robot's trajectory. Therefore, continuous connectivity can be achieved by exploiting the diversity through signal combining [31]. It was furthermore found that widening the angle of emission at the transmitter and the field of view (FOV) at the receiver is highly effective to render the link changes smoother during motion [32]. Because widening of the transmit angle leads to a reduction of the optical intensity and hence signal-to-noise-ratio (SNR) at the receiver, low SNR operation was identified as an important design goal for an optimized physical layer (PHY). A first proof-of-concept was set up with the goal to demonstrate realistic data transfer. The system involved a commercially available chipset with a modern PHY based on adaptive DCO-OFDM and low-density-parity-check (LDPC)-codes, compliant with the ITU-T G.9960 recommendation [33]. Redundant transmission and equal gain combining at the receiver were implemented to exploit the spatial diversity between the $8 \times 6$ transceivers. The results confirmed that the fast-moving industrial robot could be connected reliably and continuous connectivity with low latencies is feasible [34].

\section{Contribution and Paper Structure}

The next step is to supply a whole factory hall with LiFi. To cover large areas and serve many devices in parallel, the LiFi network needs to become scalable through combining the spatial diversity for robustness with spatial multiplexing.

The objective of this paper is to develop a system concept for industrial $\mathrm{LiFi}$ communication networks that cover large areas, support mobility, and fulfill the high requirements on QoS in industrial communication through dynamically combining spatial diversity and multiplexing. Compared to [35], we present additional background from the literature, describe the proposed system in more detail, and report recent results on several key aspects.

This article is structured as follows: In Section II, we elaborate the requirements and challenges for an industrial LiFi network. In Section III, we describe the proposed system concept. In Section IV, we present first system level simulations of the proposed medium access control (MAC) protocol. Implementation of the industrial LiFi system is discussed in Section V. As such, we present experiments on multiuser MIMO precoding, a simulative evaluation of our low-power PHY design, and our experiments with plastic optical fiber (POF) for analog fronthaul. We also discuss possible network integration approaches. Finally, we provide insights into ongoing standardization in Section VI and conclude with a summary in Section VII.

\section{REQUIREMENTS AND CHALLENGES FOR INDUSTRIAL LIFI}

An industrial LiFi network should provide sufficient performance, i.e., QoS in terms of end-to-end throughput, latency, and reliability, to support as many existing industrial applications as possible. However, it is not clear whether demanding class $\mathrm{C}$ applications like control loops will be realized for mobile users in the near future. Instead of aiming to reach performance equal to wired technologies, industrial $\mathrm{LiFi}$ should thus create value through its unique selling points and populate the gap between radio communication and wired technologies. Therefore, we assume that latencies, jitter, and reliability of industrial LiFi systems should first aim for class B performance in order to support factory automation tasks, supporting class $\mathrm{C}$ performance as far as possible. On the other hand, LiFi networks should also allow scaling up the number of users and traffic streams while supporting seamless connectivity for moving users such as robots. Furthermore, LiFi should provide additional value through support of positioning and a higher level of security to drive new and innovative developments in the industrial IoT. Typical production facilities are already equipped with a range of radio-based communication systems. While coexistence between radio and LiFi is naturally given based on orthogonal spectrum, an industrial LiFi network should actively support interworking with radio networks. This includes handing a user's connectivity over between the two networks or even facilitating the combined use of both with the goal to increase end-to-end reliability through redundant transmission over multiple media.

Albeit LiFi has properties that are very suitable for industrial communication its directionality and the inability of light to penetrate opaque objects also create new challenges for system design. To achieve high availability, for example, it is crucial that LiFi systems are able to tolerate sudden blockage of the LOSs so that connectivity is maintained. Because each $\mathrm{LiFi}$ transceiver covers only a few $\mathrm{m}^{2}$, many transceivers must be deployed in order to supply a larger area. This results in the need for frequent handovers of moving users between the luminaires, adding protocol complexity and potential outage during handover events. Due to the beam characteristics of emitters and receivers, the optical signal strength depends on the angles of emission and incidence. As a result, the SNR for users is lower at the cell edge. For moving users, the link capacity varies within the coverage area, making it difficult to provide guaranteed data rates for traffic with high QoS requirements. We conclude that a new system design approach is necessary in order to fulfill the high requirements on QoS in industrial applications.

\section{DISTRIBUTED MIMO FOR INDUSTRIAL LIFI}

In [35], we proposed to realize industrial $\mathrm{LiFi}$ based on a large scale distributed multiuser MIMO approach in order to cope with the distinctive characteristics of light as a medium. Distributed MIMO allows to implement spatial diversity and 
spatial multiplexing, and makes mobile LiFi communication with industrial-grade QoS possible.

In traditional MIMO links, at most a few antennas are supported by each access point (AP). In order to support mobility within a larger coverage area, handovers between multiple APs are needed. Handovers are well known to have critical impact on latency and reliability. A key aspect of our proposal is to scale up the number of distributed OFEs that are controlled by a single AP so that it can cover large areas. As a result, moving users stay connected as the AP dynamically selects the right set of OFEs. This enables virtually seamless connectivity without the need for handover protocol procedures. Moreover, centralized signal processing for the distributed OFEs facilitates the use of MIMO schemes to increase link robustness and throughput further in order to deliver a consistently high QoS, which is necessary for industrial communications.

\section{A. Distributed MIMO}

Contrary to traditional MIMO, where all antennas are colocated at one device, distributed MIMO denotes an architecture in which antennas are spatially separated, e.g., in LiFi-capable luminaires spread-out across the ceiling. Recent literature investigated various aspects of distributed MIMO for LiFi. For example, the authors of [36] and [37] investigate the performance of different MIMO schemes, such as repetition coding, spatial multiplexing, and spatial modulation for distributed MIMO with a single user.

Serving multiple users in a single MIMO transmission is called multiuser MIMO [38]. The idea of multiuser MIMO is that multiple spatial streams transport data for different users. The authors of [39] and [40] investigate block-diagonalization and zero-forcing precoding schemes for multiuser MIMO LiFi. In [41], the design of a transceiver for multiuser MIMO is presented. Moreover, many aspects of distributed multiuser MIMO were investigated for radio communications by the $3 \mathrm{rd}$ generation partnership project (3GPP), where the technology is known as coordinated multi-point (CoMP). Following fundamental ideas in [42], the whole cellular network, which consists of many base stations and users, can be regarded as a huge, distributed MIMO link. By joint transmission and detection, the interference in a cellular system can be converted into useful signal, given that the wireless infrastructure knows the whole MIMO channel. The gains in CoMP systems are potentially huge, particularly at the cell-edge where the coverage areas of adjacent base stations overlap. However, implementation turned out to be a challenge due to several aspects. For example, the distributed base stations require highly precise synchronization and the delay between channel estimation, feedback, and data transmission leads to outdated channel state information (CSI) at the precoder. Complexity was identified as the main problem of CoMP and it is commonly considered manageable in a new architecture denoted as Cloud-RAN [43], [44]. The Cloud-RAN architecture differs from previous architectures through the heavy use of centralized signal processing [45].

\section{B. Centralized Processing Concept}

To reduce the complexity and enable tight coordination of MIMO transmissions from different points, the proposed system performs joint processing for all distributed OFEs within a single AP. Such APs are therefore referred to as central units (CUs), see Fig. 1. Luminaires that are equipped with distributed OFEs are from now on denoted as distributed units (DUs). Each DU can cover a certain area with its light cone and the cones of neighboring DUs overlap to provide homogenous coverage. A fronthaul network connects all DUs with the CU. Consequently, DUs are understood as antennas of the CU, which can use them to transmit and receive signals to and from mobile units (MUs), respectively. Because a CU coordinates both multiple access and MIMO precoding for all DUs and MUs in the whole coverage area, no exchange of information between multiple APs is necessary. Mobility is supported by selecting suitable DUs for each MU based on the channel state, which is equivalent to antenna-selection in radio systems when using an antenna array. Multiple ways to split functionality between the CU and DUs are conceivable. The approach to place certain functional blocks either in the $\mathrm{CU}$ or in the DUs is known as functional split in 3GPP networks [46]. For example, the CU may operate on analog signals while DUs perform the conversion between the electrical and the optical signal. In another extreme, DUs may receive MAC frames from the $\mathrm{CU}$ and perform timed medium access and PHY processing in the downlink, as well as demodulation and decoding of frames in the uplink. The latter comes with reduced possibilities for joint reception in the uplink, however. Potential technologies for the fronthaul cabling include coaxial cables, twisted pair cables, and POF to transport the analog baseband signals. Digital networks like Ethernet and common public radio interface (CPRI) [47] are able to transport user data or digital signal samples. In Section V.C, we present recent work on a POF-based fronthaul link for industrial environments.

\section{Feedback Protocol for Centralized Control}

To select the best DUs based on the MU's mobility, a scheduler in the $\mathrm{CU}$ must consider the latest channel state. Moreover, the modulation and coding scheme (MCS), i.e., rate, must be selected carefully such that frame losses and increased latencies through retransmission are avoided. To obtain the necessary information for this scheduling, the CU assesses the channel between all its DUs and the MUs periodically. The basic sequence of this control protocol is depicted in Fig. 2 and involves the following three frame exchanges:

a) Downlink transmission of MIMO pilots

b) Feedback of the measured downlink CSI to the CU

c) Update of resource allocations for uplink transmissions

At time 1, upon reception of the first downlink frame, all MUs estimate the CSI towards the DUs based on the received MIMO pilot signals. The CSI is then reported to the $\mathrm{CU}$ in a feedback frame. Besides the reception of the feedback frames, the uplink channel of the transmitting MU is measured at the receiving DUs at time 2 . With the gathered channel knowledge, 


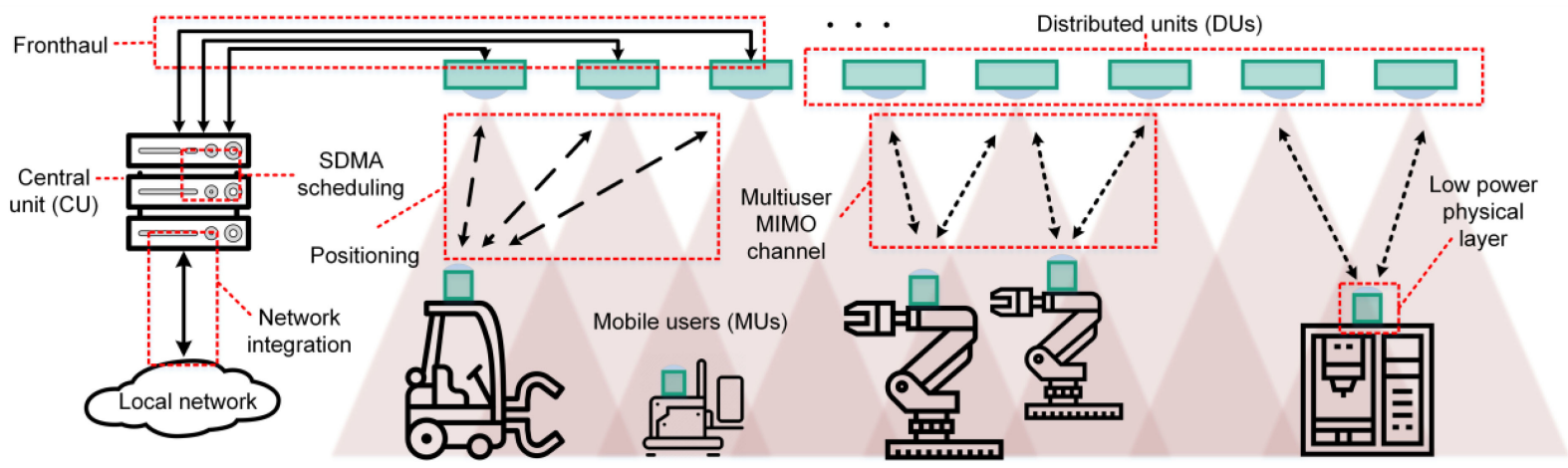

Fig. 1. The distributed multiuser MIMO system architecture, depicting a CU connected with DUs via a fronthaul network. Mobile and stationary devices communicate via the LiFi infrastructure at the same time.

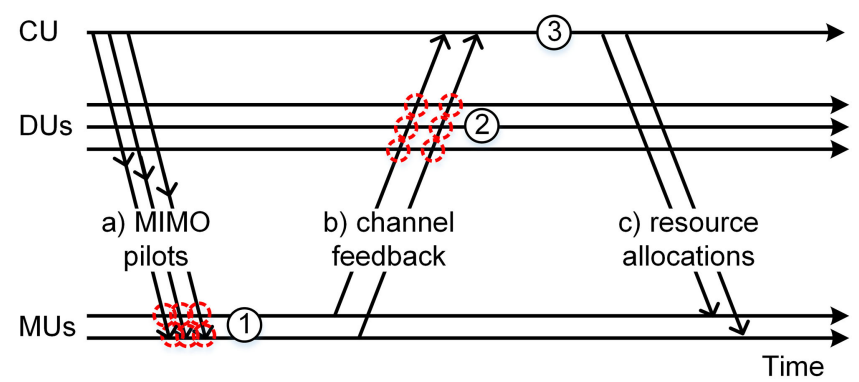

Fig. 2. Basic MAC protocol for distributed multiuser MIMO: DUs transmit MIMO pilots, which are received by MUs at time 1 . Red rings indicate channel measurement. MUs send the obtained CSI to the CU and uplink channels are measured at time 2. Based on the CSI, the $\mathrm{CU}$ schedules access and transmits resource allocations to the MUs at time 3 .

the $\mathrm{CU}$ schedules the medium access at time 3. Therefore, it allocates resources for multiplexing and joint transmission such that interference is ruled out. Subsequently, the CU distributes resource allocations for transmissions of MUs through resource allocation frames.

\section{Multiuser Access and Scheduling}

Distributed medium access schemes often rely on sensing whether the channel is clear before starting a transmission. If the channel is busy, the transmitter waits until the channel is clear. The fundamental assumption is that the channel is clear at the receiver if it is clear at the transmitter. This is not reliable with the directional propagation of light. The resulting invisibility of other transmitters is known as hidden terminal problem and results in frequent collisions.

To avoid hidden terminal problems in the first place, the proposed system relies on centrally scheduled medium access for all transmissions. Moreover, centralized scheduling allows to reserve resources for real-time transmissions. A simple and wellknown deterministic scheme is time-division multiple access (TDMA), where all MUs receive and transmit data sequentially in dedicated time slots. Generally, simultaneous transmissions are possible in orthogonal resources, e.g., different spaces or frequency bands. They improve the support for industrial communication because multiple real-time traffic streams with fixed cycle times can be transmitted in parallel without sharing transmission time. A core objective of the proposed MAC protocol is the additional support for spatial multiplexing. Therefore, we focus on space division multiple access (SDMA), where the spatial domain is represented by DUs over which different data streams can be transmitted in parallel. The CU's scheduler dynamically allocates time slots and DUs to the MUs based on the latest reported CSI. Thus, the available resources for the coordinated LiFi network may be understood as a grid of DUs $x$ time slots. The scheduler operates on this $2 \mathrm{D}$ resource grid to find the best tradeoff between diversity and multiplexing [48]. The fundamental issue is to decide whether to allocate more DUs to a MU for increased diversity or less in order to allow simultaneous transmissions for multiple MUs. Such decisions should be based on the throughput and reliability requirements of individual traffic streams. Suitable scheduling algorithms are subject to further research. Additional multiuser MIMO precoding of transmissions from multiple DUs can optionally be applied to reduce interference and improve the throughput. For multiuser MIMO with precoding, each MU still requires only a single photodiode. In Section V.A, we elaborate on multiuser MIMO precoding for interference reduction.

The described concept is agnostic with respect to the applied duplex scheme. However, simultaneous transmissions in the uplink and downlink are beneficial to reduce latencies. This may be realized, e.g., by using different wavelengths for each link direction. With time-division duplex, traffic needs to be queued until the next transmission opportunity in the desired direction. On the other hand, time-division duplex reduces complexity at the OFE, as (electrical) crosstalk between the transmitter and receiver can be ignored.

\section{E. Physical Layer (PHY)}

The PHY defines the transmitted waveform, including error coding and modulation, and aids the MAC through measuring the channel. We propose to make use of different PHYs for downlink and uplink transmissions. Both are chosen so that they support the different requirements for downlink and uplink in an optimal way. Because MUs may be battery powered, the uplink PHY should be energy-efficient and able to operate at a low SNR. One approach is to apply on-off-keying (OOK) in the 
uplink, as we detail in Section V.B. In the downlink, signals are transmitted from the infrastructure and energy efficiency at the transmitter is less relevant. Thus, we propose to aim for high spectral efficiency using DCO-OFDM with a powerful forward error correction scheme. To support the channel measurement and feedback protocol proposed in Section III.C, both PHYs must support suitable MIMO pilots. Based on these pilots, the uplink and downlink MIMO CSI between all DUs and MUs can be measured at reception and fed back to the $\mathrm{CU}$ for scheduling. Frames that are transmitted in the downlink from multiple DUs simultaneously include pilots that are orthogonal, e.g., in the time, frequency or code domain. Since we do not consider MIMO processing in the uplink yet, it is sufficient if MUs transmit in orthogonal timeslots, so that the receiving DUs can measure the uplink channel, e.g., based on the preamble of the PHY frame.

\section{F. Integrated Positioning}

Accurate positioning is considered an enabling feature for wireless communication in factories, e.g., to locate automated guided vehicles [49]. Typically, positioning algorithms use information about the signal strength, the angle, or the distance, i.e., the propagation time, between the anchor points and the MU. The authors of [50], [51], and [52] consider approaches based on signal strength, whereas [53], [54], and [55] describe approaches based on the propagation time. LiFi comes with good preconditions as a positioning technology due to the dominant LOS propagation. Moreover, the high bandwidth increases the accuracy of timing information that can be derived from the signal's phase.

We propose to realize positioning based on multilateration, operating on time-of-flight measurements between a MU and several DUs. For this scheme, a single photodiode at each MU is sufficient. Most importantly, however, the fine-granular information about the time-of-flight can be derived from the CSI that is already available through the feedback procedure presented in Section III.C. This allows for an efficient implementation as a piggyback on the ordinary network operation. To measure the distances between DUs and MUs, a coarse timestamp is obtained at the transmission and reception of frames at the DUs and the MUs. This timing information is then additionally refined based on phase information of the intensity-modulated signal, which is part of the MIMO CSI. The MAC protocol requires only minor adaptations in order to integrate positioning. In this way, real-time position information can be obtained with a high update rate, since channel information is already exchanged frequently in order to support mobility and link adaptation. The added value of positioning besides communication by using the same infrastructure and mobile devices will lighten the adoption by customers and increase the return-on-invest when installing LiFi for industrial applications.

\section{System LeVel Simulations}

To validate the proposed system and MAC protocol, we performed initial system level simulations. Considering LOS transmission, channels and PHYs are comparable with those in non-industrial scenarios. At the protocol level, however, the focus is on how the system supports industrial requirements such as homogenous coverage and reliability and not necessarily high throughput. Therefore, we developed a new simulation model for the network simulator ns-3 [56]. ns-3 is a discrete event-based network simulator, which includes support for many existing technologies such as LTE, Wi-Fi, and widely used networkand transport layer protocols. Previously, the authors of [57] implemented an OWC model for ns-3 and validated it against experiments. Our new model was developed independently and focuses on the protocol level aspects of the proposed distributed MIMO architecture.

\section{A. Simulation Model}

We implemented new models for the channel, the PHY, and the distributed multiuser MIMO MAC. In the channel model, we consider only LOS propagation, as reflections were shown to play a minor role if a wide-beam transmitter and a wide FOV receiver point downwards and upwards, respectively. We calculate the gain at the transmitter based on a Lambertian emission characteristic as

$$
G_{\mathrm{TX}}=(m+1) \cos ^{m}(\Phi)
$$

where $m$ is the Lambert order of the light emitter, and $\Phi$ the angle of emission. The receiver gain is calculated as

$$
G_{\mathrm{RX}}=\left\{\begin{array}{ll}
A_{\mathrm{PD}} \cos (\phi) R g_{\mathrm{C}}(\phi) g_{\mathrm{F}}, & |\phi| \leq \phi_{\mathrm{FOV}} \\
0, & |\phi|>\phi_{\mathrm{FOV}}
\end{array},\right.
$$

where $A_{P D}$ is the area of the photodiode, $\phi$ is the angle of incidence, $R$ is the photodiode responsivity, and $g_{\mathrm{C}}$ and $g_{\mathrm{F}}$ are the concentrator and filter gains respectively. The value of $\phi_{\mathrm{FOV}}$ denotes the FOV of the receiver. The geometric path loss is furthermore

$$
H_{\mathrm{PL}}=\frac{1}{2 \pi \cdot d^{2}} .
$$

Consequently, the optical LOS-gain between a transmitter and receiver can be written as

$$
H=G_{\mathrm{TX}} \cdot H_{\mathrm{PL}} \cdot G_{\mathrm{RX}} \cdot
$$

From the LOS gain and the positions of transmitter and receiver, we can obtain the impulse response for each transmission by assuming light speed propagation. For received signals, we calculate the signal to interference plus noise ratio (SINR) as follows: The photodiode sums all received optical power that originates from the set $S$ of serving DUs and converts it jointly into an electrical signal power. Moreover, there are interfering optical signals from the set $I$ of other active DUs, which are summed up as well before converting them into the electrical interference power. Finally, we assume additive white Gaussian noise, such that the effective SINR is calculated as

$$
S I N R_{\mathrm{eff}}=\frac{\left(\sum_{s \in S} H_{\mathrm{s}} P_{\mathrm{s}}\right)^{2}}{\left(\sum_{\mathrm{i} \in \mathrm{I}} H_{\mathrm{i}} P_{\mathrm{i}}\right)^{2}+\sigma^{2}} .
$$

Here, $H_{\mathrm{S}}, H_{\mathrm{I}}, P_{\mathrm{S}}$ and $P_{\mathrm{I}}$ are the LOS channel gain between the serving transmitters and the receiver, the gain between interfering transmitters and the receiver, and the optical powers of 
TABLE II

EsSEnTial Simulation Model Parameters

\begin{tabular}{ll|ll}
\hline \hline \multicolumn{1}{c|}{ Parameter } & \multicolumn{1}{c}{ Value } & \multicolumn{1}{c}{ Parameter } & \multicolumn{1}{c}{ Value } \\
\hline LED Lambert order & 1 & Filter gain & 1 \\
Electrical transmit power & $35 \mathrm{dBm}$ & Concentrator gain & 1 \\
Photodiode responsivity & $0.28 \mathrm{~A} / \mathrm{W}$ & Electrical noise & $-92.2 \mathrm{dBm}$ \\
Photodiode area & $1 \mathrm{~cm}^{2}$ & PHY Bandwidth & $100 \mathrm{MHz}$ \\
\hline \hline
\end{tabular}

serving and interfering transmitters, respectively. $\sigma^{2}$ denotes the noise power. For simplicity, we assume that LOS signals from serving transmitters arrive simultaneously at the receiver, i.e., that no frequency-selective fading occurs through the superimposed multipath from multiple DUs.

We performed measurements with an arbitrary waveform generator and an oscilloscope to obtain realistic values for the SNR. For the constant gain factors, photodiode area, and responsivity, we adopted values from the literature. We then set the values for the transmit power, noise power, and Lambert order of the LED to match the measured SNRs in various geometric constellations. The relevant simulation parameters are listed in Table II. Note that the setup involved an OFE with wide emission angle in order to support seamless mobility as proposed in Section I.B and [32]. This is in contrast to many academic publications that consider narrow beams, which result in higher SNRs. The achievable absolute values for SNR or data rates may vary for other OFE realizations.

Our PHY model is based on DCO-OFDM with LDPC, as specified in ITU-T G.9960 [33]. The model assumes 509 usable of 512 total subcarriers over $100 \mathrm{MHz}$ of bandwidth. To simplify the simulator, we consider only uniform bit-loading and calculate a set of supported data rates based on the available MCSs. Each MCS constitutes of a modulation between binary phase shift keying (BPSK) and 256-quadrature amplitude modulation (QAM) as well as a code rate between $1 / 2$ and 20/21. The PHY is frame-based and includes a preamble, header, and payload. Transmitters remain silent if no actual PHY frame is being transmitted. The model calculates the frame duration for each transmission based on the selected MCS, payload length and preamble as well as header information. For the following simulations, we use the same PHY in the uplink and downlink.

The MAC layer model supports the essential MAC frames. That is, among others, a beacon frame for network synchronization, which contains also downlink MIMO pilots. Moreover, the model includes frames for the CSI feedback and resource allocations to support the protocol routines described in Section III.C. Data frames carry the application data. All medium access is performed in a dynamic SDMA, where the DUs and MUs transmit in time slots that are allocated by the CU's scheduler. We implement time-division-duplex, i.e., reserve half of the time slots for the downlink and uplink, respectively. The $\mathrm{CU}$ is able to start simultaneous downlink transmissions from selected DUs, whereas from the MU's perspective, uplink transmissions are just started in a specific time slot. The fronthaul is modeled as a configurable delay in the $\mu$ s range. To simulate load, we transmit a data stream from the $\mathrm{CU}$ to the $\mathrm{MU}(\mathrm{s})$. For each received frame,

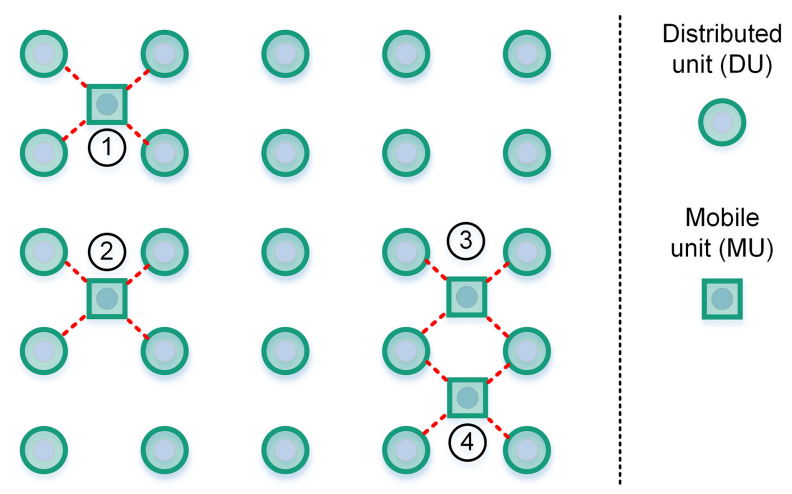

Fig. 3. Top view of five by five DUs serving four MUs. MU 1 and 2 are served by disjoint sets of DUs. MU 3 and 4 observe two same DUs. Such interference clusters are identified and the spatial contention resolved by the scheduler in the proposed industrial LiFi system.

we trace the SINR and other parameters, as calculated by the channel and PHY model.

\section{B. Baseline Scheduling Algorithm}

Our scheduler is designed to validate the basic functionality of the proposed protocol. We employ a simple scheduling algorithm, which aims to reduce interference and increase spatial diversity. These are relevant goals for industrial communication in order to realize reliable transmission. Typical performance indicators in non-industrial scenarios, like throughput or proportional fairness, are not considered. Rather, the scheduler periodically allocates time slots fairly based on the latest CSI as follows

1) Identify clusters, where each cluster includes all MUs that have an intersection in observed DUs. A MU is considered to observe a DU when the measured channel strength exceeds a configurable clustering threshold. The clustering threshold is defined with respect to the noise power. In Fig. 3, for example, three clusters could be found:

- MU 1 and observed DUs

- MU 2 and observed DUs

- MU 3 and MU 4 and their respectively observed DUs

2) Schedule MUs in each cluster independently from MUs in other clusters. Since interference between clusters is ruled out, each cluster can assign all the time slots in the scheduling period among the MUs. Multiple MUs within clusters are scheduled in a fair TDMA to prevent interference. For example, MU 3 and MU 4 in Fig. 3 are scheduled in different time slots, while MU 1 and MU 2 can reuse the same time slots in SDMA.

3) The serving DUs for each MU are selected. Theoretically, all DUs in a cluster can be allocated to each MU, as interference cannot occur. The maximum number of DUs that the scheduler allocates per MU can be limited by the max. DUs parameter. Depending on this parameter, the scheduler allocates only those DUs that have the strongest signal at the MU. Varying this parameter allows to shift the degree of multiplexing vs. diversity.

As a result of the scheduling, interference between MUs within a cluster is coordinated through orthogonal time slots. Spatial multiplexing occurs after a MU moved far enough away 


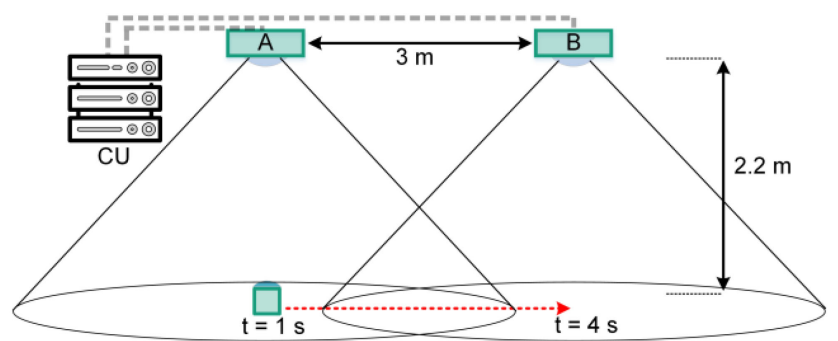

Fig. 4. Scenario 1: A single MU moves between the coverage of DU A and DU B while the CU transmits data frames in the downlink.

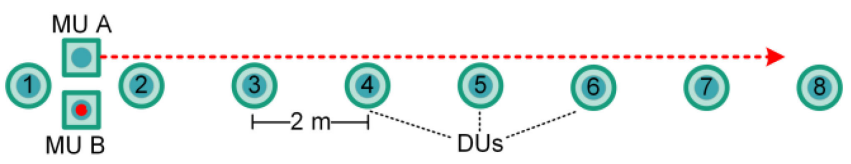

Fig. 5. Scenario 2, where MU A moves along a line of DUs and MU B remains static. Data frames are transmitted towards both MUs from the CU.

from other MUs that it is considered an independent cluster. The described SDMA scheduling can be implemented with low complexity and provides a baseline for future improvements.

\section{Scenario 1: Spatial Diversity and Soft Handover}

In the first scenario, we investigated the basic capability of the protocol to support soft mobility and spatial diversity. Between second 1 and 4 of the simulated time, the MU moves with $1 \mathrm{~m} / \mathrm{s}$ from the center of DU A's coverage to the center of DU B's coverage, as indicated through the red arrow in Fig. 4. The two DUs are controlled by a single CU and cover an overlapping region. The distance between the DUs is $3 \mathrm{~m}$ and their height is $2.2 \mathrm{~m}$ above the MU. In compliance with the proposed protocol, the $\mathrm{CU}$ transmits a beacon frame with embedded MIMO pilots through both DUs every $10 \mathrm{~ms}$. Upon its reception, the MU reports the measured signal strength, i.e., the CSI for each DU, to the CU via feedback frames. The CU's scheduler then performs a selection of the DU(s) for the MU.

We perform two simulation runs. In the first run, the scheduler always assigns at most one DU with the best signal to the MU. In the second run, the scheduler is able to assign more than one DU to the MU, resulting in joint downlink transmissions. We expect that the MU is able to receive downlink data continuously in both simulation runs. Mobility should be supported by means of dynamic DU selection, based on the CSI reported via the feedback protocol.

\section{Scenario 2: Adaptive Spatial Multiplexing}

In the second scenario, we assess adaptive spatial multiplexing applied by one CU serving two MUs. As depicted in Fig. 5, MU B has a fixed position and MU A moves along a straight trajectory, which is covered by a line of DUs. DUs reside $2.2 \mathrm{~m}$ above the MU. The space between the DUs is $2 \mathrm{~m}$ and MU A moves at a velocity of $1 \mathrm{~m} / \mathrm{s}$. Since both MUs transmit feedback frames to the $\mathrm{CU}$ periodically, the scheduler is able to determine suitable DUs to transmit to the each MU. Through identifying

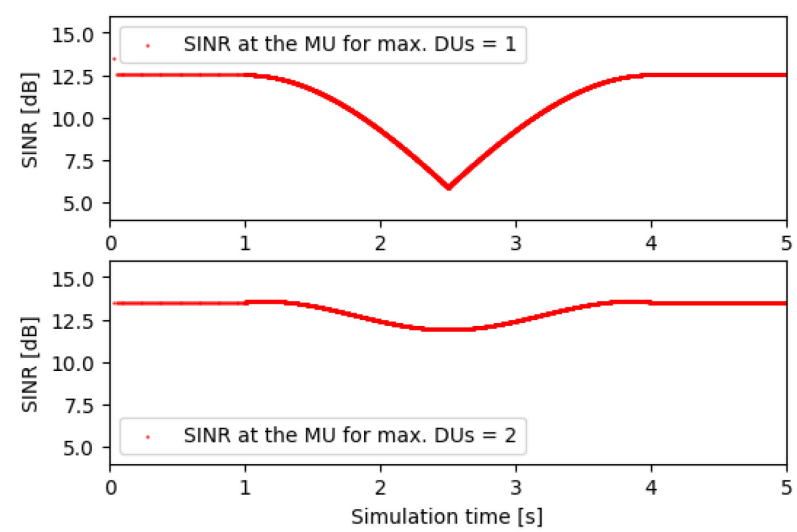

Fig. 6. Observed SINR for downlink transmissions. Frames are transmitted by the CU over the DU(s) selected by the scheduler. For the top graph, the scheduler allocates at most one DU to the MU and the other DU stays silent. In the bottom graph, two DUs were allocated to the MU, transmitting jointly.

clusters, the scheduler also detects whether both MUs can be served simultaneously through spatial multiplexing or whether they require orthogonal time slots because the interference would be too high. The scheduling algorithm is configured for a clustering threshold of $15 \mathrm{~dB}$ below the noise power. We expect the MUs to be spatially multiplexed after MU A has moved far enough so that its DUs cause only interference $15 \mathrm{~dB}$ below the noise at MU B. Consequently, MU A will be assigned to a different cluster than MU B.

\section{E. Results}

Scenario 1 We traced the observed SINRs of all frame receptions at the MU and plotted them in Fig. 6. The top graph depicts the SINR for the first simulation run, where the scheduler allocates the strongest DU to the MU. Between seconds 1 and 4 of the simulation time, the MU is moving and the SINR varies. At first, only DU A serves the MU and after second 2.5, as the scheduler detects that DU B has the best signal at the MU, it switches to DU B. As the unused DU has nothing to transmit, it remains silent and the SINR is equal to the SNR. The S(I)NR drops significantly from $12.5 \mathrm{~dB}$ below DU A to $5.9 \mathrm{~dB}$ at the cell edge, which translates into lower achievable data rates. Still, the MU is continuously connected, as the $\mathrm{CU}$ adaptively selects the serving DU.

The bottom graph shows the SINR for simulation run two, where both DUs serve the MU jointly. Even in the start and end position, under DU A and DU B respectively, the joint signal powers of two DUs lead to an SINR of $13.5 \mathrm{~dB}$, which is $1 \mathrm{~dB}$ higher than for transmissions via only a single DU. Moreover, the received SINR drops by only $0.6 \mathrm{~dB}$ to $11.9 \mathrm{~dB}$ when the $\mathrm{MU}$ is in-between the two cells at $2.5 \mathrm{~s}$ simulation time. Hence, the variance in SINR is much lower compared with simulation run 1 . This allows the use of a higher rate for transmissions at the cell edge, rendering mobility smoother and allowing a consistent performance.

Scenario 2 For the second scenario, we plotted the received downlink data rate at both MUs over simulation time in Fig. 7. At first, both MUs share the total number of available time slots and 


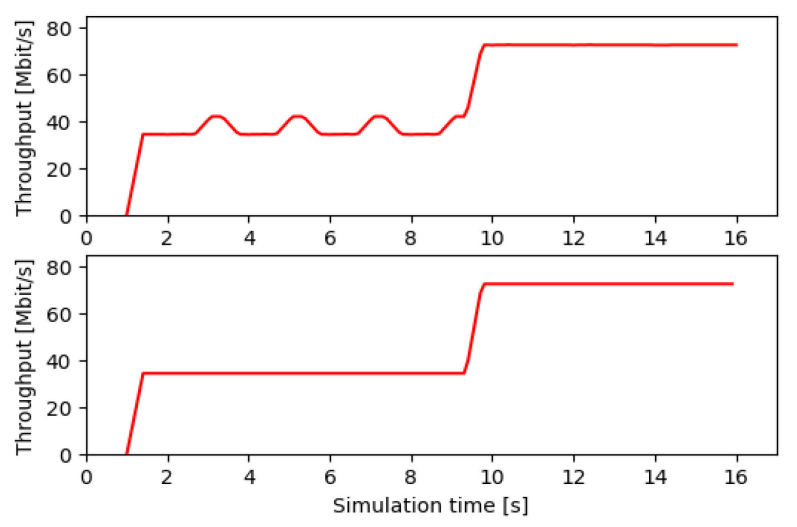

Fig. 7. The observed downlink throughput at the moving MU A (top) and the static MU B (bottom) indicates that switching between diversity and multiplexing is supported by the distributed multiuser MIMO system.

the DUs 1 to 4 are allocated to MU A and MU B. Consequently, both MUs receive with a data rate of about $38 \mathrm{Mbit} / \mathrm{s}$. The data rate was traced at the transport layer in ns-3. Each transmitted packet of 5000 bytes requires 60 bytes of protocol overhead. The selected MCS defines 16-QAM at a code rate of 2/3. The OFDM symbol duration, including a cyclic prefix, is $6.4 \mu \mathrm{s}$, which results in a PHY data rate of approx. $202 \mathrm{Mbit} / \mathrm{s}$. The duration of the PHY frame payload is therefore $191 \mu$ s. The whole PHY frame, including preamble and PHY header, requires $235 \mu$ s air time. As each transport layer packet results in a PHY frame and half of the time slots are reserved for the uplink, the available downlink data rate is around $86 \mathrm{Mbit} / \mathrm{s}$ when all available time slots are combined. The remaining overhead results from the beacon frame, uplink allocation frames and inter-frame gaps.

MU A's data rate varies as it passes the DUs and the scheduler reacts to the changes of the SINR by selecting different MCS. From second 9.3 in the simulation time, DU 4 has a signal strength of less than $15 \mathrm{~dB}$ below the noise at MU A. Based on the fed back CSI, the scheduler detects that interference is low enough to form a second cluster for MU A and allow simultaneous transmissions. Now MU A is served by DU 5 to 8 in the same time slots as MU B is served by DU 1 to 4 . As a result, the data rate of both MUs increases to around $76 \mathrm{Mbit} / \mathrm{s}$. Note that our scheduler operates very conservatively and allocates all DUs for full diversity. Allocating less DUs and allowing for more interference would have led to earlier switching to spatial multiplexing.

\section{IMPLEMENTATION AND OPTIMIZATION}

In this section, we highlight selected implementation and optimization aspects of the distributed multiuser MIMO system.

\section{A. Multiuser MIMO Precoding}

As described in Section III, the CU has a global view on the network and can coordinate the medium access in the uplink and downlink in the space and time domains. Within a spatial cluster, our scheduler in Section IV performed TDMA to coordinate interference, i.e., between MU 3 and 4 in Fig. 3. However, as

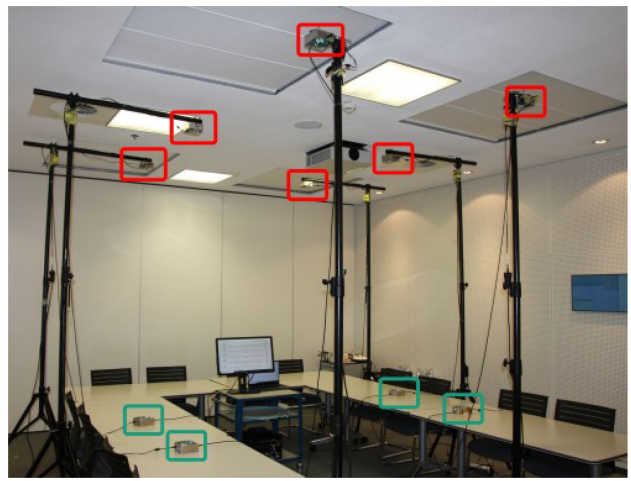

Fig. 8. Measurement setup in a conference room with six transmitters (red) at the ceiling and four receivers (green) on the tables [62].

an advanced method for interference handling, joint precoding can be applied in order to reduce the cross-talk and transmit data streams in parallel. This increases the achievable throughput and does not require additional functionality at the MU.

Precoding allows to control how the signals from different transmitters combine at multiple receivers. In [58], random grouping of users in combination with zero forcing precoding was shown to increase spectral efficiency and fairness. The authors of [59] investigated user grouping for SDMA in the multiuser MIMO downlink and confirmed that exploiting the spatial domain enables higher sum throughput for LiFi compared to orthogonal frequency division multiple access (OFDMA). However, typical distributed MIMO LiFi channels have a lower rank than comparable radio channels [23]. For a single user, it is typically proposed to increase the rank of the MIMO channel matrix through angular diversity, e.g., by using an imaging receiver [60]. To assess the potential of multiuser MIMO with and without precoding, we performed several experiments. In [61] it is shown that the capacity gain through zero-forcing precoding is factor two when serving two users in parallel in a neurosurgery room. Moreover, we highlight channel measurements for a distributed MIMO LiFi setup in a conference room [62]. Measurements were taken with OFEs designed for industrial applications with wider beam-width and higher power. Fig. 8 shows the distributed $6 \times 4$ multiuser MIMO setup in the $5.8 \mathrm{~m} \times 6.8 \mathrm{~m} \times 3 \mathrm{~m}$ room. The six transmitters were placed at $2.85 \mathrm{~m}$ height, pointing downwards and the four receivers at $0.75 \mathrm{~m}$ height, facing upwards. The receivers were placed in four different scenarios. Fig. 9 depicts the singular values of the compound channel matrix for the different measured scenarios. In scenario 1, all receivers are placed closely together. As a result, only one significant singular value can be observed, i.e., only one data stream can be transmitted and all users have to share the time domain. In scenario 2, two receivers are spatially separated and two strong singular values exist so such that two parallel data streams are possible. In scenario 3, one receiver pair was placed close to each other and the other two receivers at two different positions. Now there are three significant singular values, i.e., three parallel data streams. In scenario 4 , a distance of 2 to $3 \mathrm{~m}$ between all receivers resulted in the channel having 


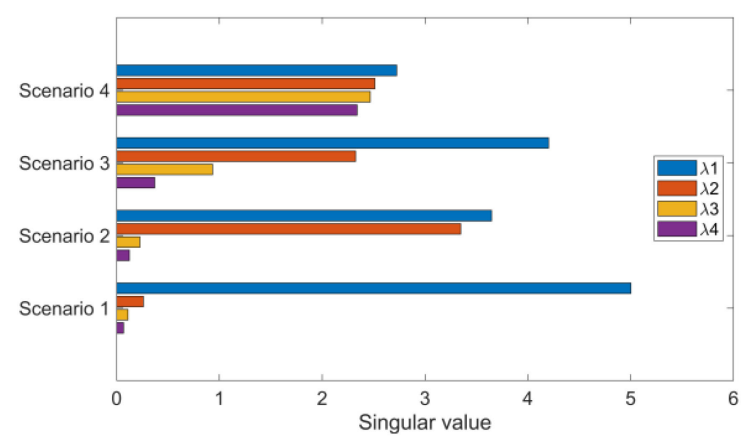

Fig. 9. The number of significant singular values increases from scenario 1-4. When users are more distant, more streams can be transmitted in parallel, indicating the benefit of spatial multiplexing [62].

four similar singular values, indicating full spatial multiplex with four parallel data streams.

These results show that spatial multiplexing is possible but requires that users are spatially separated to avoid the rank deficiency problem. We expect similar results in industrial scenarios, with an increasing probability of blockage for the LOS. The scheduler needs to know the rank of the compound multiuser MIMO channel matrix in order to make meaningful decisions. Integration of precoding in our scheduler implementation is considered future work.

\section{B. Low-Power PHY}

Because of battery powered devices and the proposed wide transmission angles, efficient use of signal power to transport information is a primary concern in industrial LiFi systems. We investigated a PHY for LiFi that relies on OOK modulation to reduce energy usage in the OFE at the expense of spectral efficiency. OOK has been a popular topic in $\mathrm{LiFi}$ research and is also investigated in [20], [63] [65], [66]. As proposed in Section III.E, such a PHY can be used in the uplink from the MU to the DU. In contrast to OFDM, the peak-to-average power ratio of OOK is one, increasing the average output power under peak-power limitations. Moreover, the amplifier in the LED driver can be operated in a switching mode, saving a significant amount of the power in the OFE [64]. Since the spectral efficiency is low, a higher bandwidth compared with OFDM-based systems is needed to achieve a given throughput. Modern LED-based OFEs can deliver bandwidths up to some $100 \mathrm{MHz}$, enabling data rates of more than $100 \mathrm{Mbit} / \mathrm{s}$ with OOK, which is sufficient for many industrial applications.

The following figures summarize evaluation results of a PHY based on OOK, 8B10B and Reed-Solomon error coding. The PHY supports block-based frequency-domain equalization for high symbol rates and is also being specified as Pulsed Modulation PHY (PM-PHY) in IEEE P802.15.13. Fig. 10 shows the detection success rates for a PHY frame's synchronization preamble, header, and payload at a symbol rate of $200 \mathrm{MHz}$, over a LOS channel with additive white Gaussian noise [64], [67]. As part of the PHY frame, the synchronization preamble serves the frame detection at the receiver, whereas the header includes information necessary for demodulation of the payload.

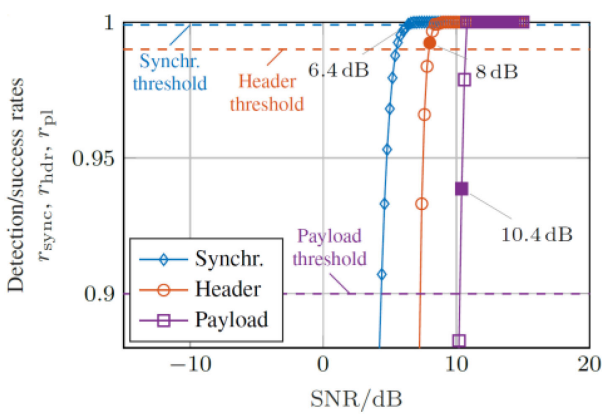

Fig. 10. Simulated success rates for the synchronization preamble, header and payload parts of the OOK PHY frame [64].

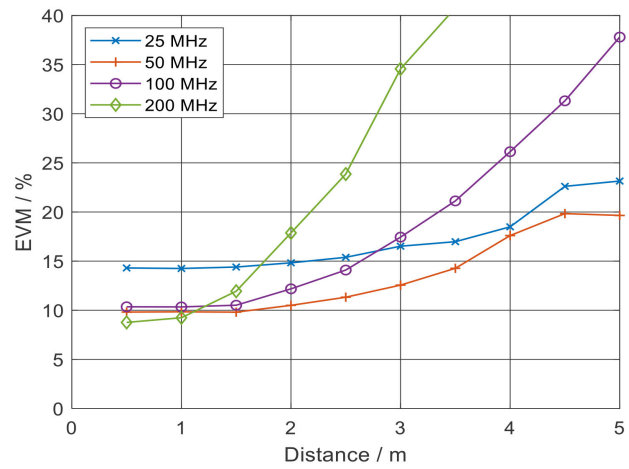

Fig. 11. Measured error vector magnitude (EVM) for different distances between a DU and a MU, using the OOK PHY at different symbol rates [68].

Therefore, all three portions must be correctly detected. We found that the PHY operates reliably starting at an SNR of $10.4 \mathrm{~dB}$, where the payload is correctly detected.

Results from experimental measurements of the error vector magnitude (EVM) in a laboratory setup are described in [68] and shown in Fig. 11. Here, the achievable distance decreases with increasing symbol rate. This is attributed to the characteristics of the used OFEs, reducing signal power at higher symbol rates. The results confirm that error-free transmission is possible at $10 \mathrm{~dB}$ SNR, corresponding to an EVM of $20 \%$, consistent with simulations as described in [68].

\section{Fronthaul Using POF}

Different network technologies may serve as fronthaul as long as they transport the signal between the CU and DUs with the required delay and signal quality. Comparing different fronthaul approaches, analog fronthaul techniques were found to be spectrally efficient [69]. In a system with analog fronthaul, all signal processing and digital-to-analog conversion is performed in the CU. Accordingly, the fronthaul transports analog signals between the CU and DUs. However, electrical analog fronthaul cables may suffer from electromagnetic interference in the baseband, e.g., due to radio broadcasting [30]. Since optical transmission is inherently robust against electromagnetic interference, $\mathrm{POF}$ is a promising fronthaul technology for industrial environments, where reliability is important and large electrical machines may produce additional emissions in the baseband. 


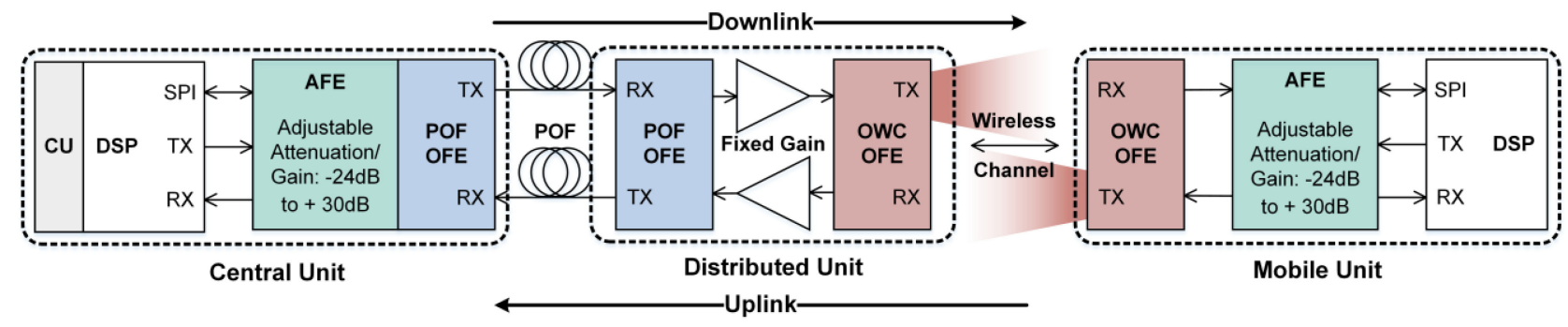

Fig. 12. Block diagram of the bidirectional POF and LiFi link between the CU and DU. A fixed gain amplifier is inserted between the POF and LiFi links [71].

TABLE III

MEASURED THROUGHPUT OF THE LIFI LINK IN DOWN- AND UPLINK WiTHOUT AND WITH POF FRONTHAUL FOR SEVERAL DiSTANCES [71].

\begin{tabular}{cc}
\hline \hline Link Configuration & Throughput Downlink / Uplink (Mbit/s) \\
\hline OWC $30 \mathrm{~cm}$ & $882 / 959$ \\
OWC $50 \mathrm{~cm}$ & $922 / 938$ \\
OWC $70 \mathrm{~cm}$ & $759 / 800$ \\
OWC $100 \mathrm{~cm}$ & $681 / 743$ \\
OWC $130 \mathrm{~cm}$ & $596 / 671$ \\
OWC $150 \mathrm{~cm}$ & $470 / 611$ \\
POF $10 \mathrm{~m}+$ OWC $30 \mathrm{~cm}$ & $725 / 901$ \\
POF $10 \mathrm{~m}+$ OWC $50 \mathrm{~cm}$ & $537 / 604$ \\
POF $10 \mathrm{~m}+$ OWC $70 \mathrm{~cm}$ & $446 / 435$ \\
POF $10 \mathrm{~m}+$ OWC $70 \mathrm{~cm}$ & $313 / 218$ \\
POF $10 \mathrm{~m}+$ OWC $130 \mathrm{~cm}$ & $239 / 145$ \\
POF $10 \mathrm{~m}+$ OWC $150 \mathrm{~cm}$ & $218 / 115$ \\
\hline \hline
\end{tabular}

In [70], the bandwidth and capacity of a single link was measured to demonstrate the feasibility of POF as fronthaul for LiFi. We implemented a bidirectional fronthaul link to cover the required dynamic range of the wireless path and the wired link, as shown in Fig. 12 [71]. Using POF to transport analog signals between the $\mathrm{CU}$ and DUs without digital signal regeneration revealed a static loss depending on the length of the POF and a dynamic loss of up to $20 \mathrm{~dB}$. In the downlink, the dynamic range can be compensated through an automatic gain control (AGC) in the MU, consisting of a variable gain amplifier (VGA) in the analog frontend (AFE), being controlled by the co-located digital signal processor (DSP). In the uplink direction, on the other hand, the DSP is located in the CU. Remote control of the VGA in the DU's OFE is challenging. Therefore, we compensated the loss over the POF by a fixed-gain amplifier and placed the VGA at the CU. Table III lists measured throughputs for several link configurations. The results show a drop of the data rate due to non-ideal compensation of the dynamic range, which becomes significant for larger wireless distances. Over $10 \mathrm{~m} \mathrm{POF}$ and a wireless distance of $150 \mathrm{~cm}, 218$ and $115 \mathrm{Mbit} / \mathrm{s}$ could be achieved in the down- and uplink, respectively. In factory halls, the wireless distance may be longer.

\section{Radio Network Integration}

Unlike LiFi signals, which mainly propagate through the LOS, radio waves propagate mostly through multiple reflections, which enables connectivity in locations where the LOS is not available. Thus, radio complements $\mathrm{LiFi}$ in locations where $\mathrm{LiFi}$ signals do not propagate. On the other hand, radio can offload data to LiFi where it is available, releasing capacity on radio frequencies. In industrial networks, traffic can be partitioned between radio and LiFi links, based on its QoS requirements [72]. Moreover, redundancy over two independent media can increase the reliability for critical traffic, such as safety alerts.

Widely used radio technologies are Wi-Fi and 3GPP cellular networks, i.e., 4G/5G. Generally, integration of radio and $\mathrm{LiFi}$ can be loose or tight. By supporting layer-2, i.e., local area network (LAN), connectivity, LiFi networks can interwork with Ethernet and Wi-Fi through bridging. For a dynamic handover, the IEEE 1905.1 standard or software defined networking can be leveraged. Tight integration is achieved by the interworking of protocols on the lower layers. For example, it was proposed to extend the fast session transfer functionality of IEEE 802.11 to support LiFi besides $2.4,5$ and $60 \mathrm{GHz}$.

$5 \mathrm{G}$ networks are based on internet protocol (IP) connectivity. Offloading traffic to other access technologies is well-supported in cellular networks and 3GPP has multiple ways of integration. For example, the non-3GPP interworking function (N3IWF) describes an interface, which allows association with the core network for devices that have IP connectivity over a non-3GPP technology, e.g., Wi-Fi [10]. Similar to integrating Wi-Fi, LiFi can be integrated over an IP connection. The integration on the data plane can leverage existing standards and interfaces, whereas intelligent vertical handover as well as traffic partitioning are yet to be implemented.

\section{THE IEEE P802.15.13 PROJECT}

There are multiple activities to standardize LiFi. In 2011, the IEEE Standards Association published the IEEE Std 802.15.7. Recently, the ITU-T published G.9991 [25] as an extension to the home networking recommendation G.9960 [33]. The IEEE P802.11bb task group works on adding a new PHY for light communication to the IEEE 802.11 standard.

The IEEE P802.15.13 project is an outstanding activity among these, as it describes a dedicated standard for LiFi in industrial applications where for the first time scalable distributed multiuser MIMO operation is specified. The standard provides compatibility with the IEEE 802 family of standards and LANs, in order to facilitate interoperation with existing Ethernet-based industrial communication protocols and time-sensitive networking (TSN) in the future. The current draft defines medium access based on dynamic TDMA and SDMA using a superframe, which enables deterministic channel access through time slot reservations for users. This allows bounded and predictable latencies with small jitter, as needed 
for industrial communication. For scheduled access, each node maintains a network-wide reference time, based on superframe and slot numbers. The network time is used to time-trigger transmissions in reserved slots and may be used to distribute time synchronization across the LiFi network in the future.

The standard specifies PHYs for high throughput and energy efficiency, based on DCO-OFDM and OOK (see Section V.B). Multiuser MIMO is supported by both PHYs through the definition of MIMO pilot signals. In the downlink, these pilots are constructed from orthogonal combs of subcarriers in the frequency domain where one is used for each DU, allowing to simultaneously estimate the channels from multiple DUs at the MU. In the uplink, orthogonal binary sequences are used that are defined in the time domain. Furthermore, the draft includes the definition of MAC frames for feedback of MIMO CSI between all DUs and MUs to the CU, as described in Section III.C. The feedback contains the amplitude and delay for each link between the DUs and the MU. Spatial multiplexing is possible as the standard allows allocating the same time slots to multiple users in parallel, as long as interference can be ruled out. The current draft does not specify any MIMO precoding schemes but multiuser MIMO precoding techniques that are transparent to the user can be realized by implementers based on the CSI feedback mechanism. The current draft of IEEE P802.15.13 specifies all essential means to realize a distributed multiuser MIMO network for $\mathrm{LiFi}$ in a minimalistic manner, thus serving as a basic specification of the mandatory features for a future industrial LiFi system.

\section{SUMMARY}

In this article, we highlighted the potential of using LiFi for industrial wireless communications and outlined the requirements and challenges associated with this use case. Based on the key performance indicators, which are reliability and low latency besides moderate user data rates, we proposed to cover a manufacturing hall with an industrial LiFi network using a distributed multiuser multiple-input multiple-output architecture with centralized control. We demonstrated that this approach is capable of handling the distinctive challenges of light as a medium for high quality of service in an industrial scenario. We proposed space division multiple access as medium access scheme and pointed out that its performance can be further improved through joint precoding which cancels residual interference. We argued that the multiuser MIMO not only brings much higher quality of service into LiFi networks, but also supports integrated positioning based on time-of-flight measurements. We proposed a low-power PHY based on on-off-keying with frequency-domain equalization for battery-powered mobile devices. Moreover, plastic-optical fiber was considered as a promising fronthaul technology carrying analog signals between the central and the distributed units. We discussed the value of integration with radio technologies besides several promising approaches how to implement it. The main features described in this paper have been specified in the draft of the IEEE Std 802.15.13, which is validated through several evaluation results shown in this paper. Our next step is to implement essential features in a working prototype, overcome additional impairments and test it in a real industrial scenario.

\section{REFERENCES}

[1] H. Lasi, P. Fettke, H.-G. Kemper, T. Feld, and M. Hoffmann, "Industry 4.0," Bus. Inf. Syst. Eng., vol. 6, no. 4, pp. 239-242, Aug. 2014.

[2] B. Chen, J. Wan, L. Shu, P. Li, M. Mukherjee, and B. Yin, "Smart factory of industry 4.0: Key technologies, application case, and challenges," IEEE Access, vol. 6, pp. 6505-6519, Dec. 2018.

[3] M. Wollschlaeger, T. Sauter, and J. Jasperneite, "The future of industrial communication: Automation networks in the era of the Internet of Things and industry 4.0," IEEE Ind. Electron. Mag., vol. 11, no. 1, pp. 17-27, Mar. 2017.

[4] X. Li, D. Li, J. Wan, A. V. Vasilakos, C.-F. Lai, and S. Wang, "A review of industrial wireless networks in the context of industry 4.0," Wireless Netw., vol. 23, no. 1, pp. 23-41, Jan. 2017.

[5] S. Petersen and S. Carlsen, "WirelessHART versus ISA100.11a: The format war hits the factory floor," IEEE Ind. Electron. Mag., vol. 5, no. 4, pp. 23-34, Dec. 2011.

[6] N. Baker, "ZigBee and bluetooth strengths and weaknesses for industrial applications," Comput. Control Eng. J., vol. 16, no. 2, pp. 20-25, Apr. 2005.

[7] L. Leonardi, G. Patti, and L. L. Bello, "Multi-hop real-time communications over bluetooth low energy industrial wireless mesh networks," IEEE Access, vol. 6, pp. 26505-26519, May 2018.

[8] K. Das and P. Havinga, "Evaluation of DECT for low latency real-time industrial control networks," in IEEE Int. Conf. Sens., Commun. Netw., Jun. 2013, pp. 10-17.

[9] W. Haerick and M. Gupta, "5G and the factories of the future," 5G-PPP White Paper, 2015, Accessed: Feb. 2021. [Online]. Available: https://5g-ppp.eu/wp-content/uploads/2014/02/5G-PPP-White-Paperon-Factories-of-the-Future-Vertical-Sector.pdf

[10] A. Aijaz, "Private 5G: The future of industrial wireless," IEEE Ind. Electron. Mag., vol. 14, no. 4, pp. 136-145, Dec. 2020, doi 10.1109/MIE.2020.3004975.

[11] S. Gangakhedkar, H. Cao, A. R. Ali, K. Ganesan, M. Gharba, and J. Eichinger, "Use cases, requirements, and challenges of $5 \mathrm{G}$ communication for industrial automation," in Proc. IEEE Int. Conf. Commun. Workshops, May 2018, pp. 1-6.

[12] M. Düngen et al., "Channel measurement campaigns for wireless industria automation," Automatisierungstechnik, vol. 67, no. 1, pp. 7-28, Jan. 2019

[13] M. Weiner, M. Jorgovanovic, A. Sahai, and B. Nikolié, "Design of a lowlatency, high-reliability wireless communication system for control applications," in Proc. IEEE Int. Conf. Commun., Jun. 2014, pp. 3829-3835.

[14] A. Willig, "Redundancy concepts to increase transmission reliability in wireless industrial LANs," IEEE Trans. Ind. Informat., vol. 1, no. 3, pp. 173-182, Aug. 2005.

[15] M. Schneider, J. Rambach, and D. Stricker, "Augmented reality based on edge computing using the example of remote live support," in Proc. IEEE Int. Conf. Ind. Technol., Mar. 2017, pp. 1277-1282.

[16] G. Mohanarajah, D. Hunziker, R. D'Andrea, and M. Waibel, "Rapyuta: A cloud robotics platform," IEEE Trans. Autom. Sci. Eng., vol. 12, no. 2, pp. 481-493, Apr. 2015.

[17] S. Mumtaz, A. Alsohaily, Z. Pang, A. Rayes, K. F. Tsang, and J. Rodriguez, "Massive Internet of Things for industrial applications: Addressing wireless IIoT connectivity challenges and ecosystem fragmentation," IEEE Ind. Electron. Mag., vol. 11, no. 1, pp. 28-33, Mar. 2017.

[18] K. Govindaraj, D. Grewe, A. Artemenko, and A. Kirstaedter, "Towards zero factory downtime: Edge computing and SDN as enabling technologies," in Proc. 14th Int. Conf. Wireless Mobile Comput., Netw. Commun., Oct. 2018 , pp. 285-290.

[19] S. Melnyk et al., "Next generation industrial radio LAN for tactile and safety applications," VDE/ITG Fachtagung Mobilkommunikation, vol. 22, no. 9-10, p. 7, 2017.

[20] F. R. Gfeller and U. Bapst, "Wireless in-house data communication via diffuse infrared radiation," Proc. IEEE, vol. 67, no. 11, pp. 1474-1486, Nov. 1979.

[21] N. Serafimovski et al., "An overview on high speed optical wireless/light communications," Accessed: Feb. 2021. [Online]. Available: https://ieee802.org/802_tutorials/2017-07/11-17-0962-03-001c-AnOverview-on-High-Speed-Optical-Wireless-Light.pdf

[22] M.Z. Chowdhury, M. Shahjalal, M. K. Hasan, and Y. M. Jang, "The role of optical wireless communication technologies in 5G/6G and IoT solutions: Prospects, directions, and challenges," Appl. Sci., vol. 9, no. 20, Jan. 2019, Art. no. 20. 
[23] L. Zeng et al., "High data rate multiple input multiple output (MIMO) optical wireless communications using white led lighting," IEEE J. Sel. Areas Commun., vol. 27, no. 9, pp. 1654-1662, Dec. 2009.

[24] P. W. Berenguer, V. Jungnickel, and J. K. Fischer, "The benefit of frequency-selective rate adaptation for optical wireless communications," in Proc. 10th Int. Symp. Commun. Syst., Netw. Digit. Signal Process., Jul. 2016, pp. 1-6.

[25] High-speed indoor visible light communication transceiver, "System architecture, physical layer, and data link layer specification," ITU-T Rec. G.9991 Amendment 1, Jul. 2020.

[26] X. Liu, X. Wei, L. Guo, Y. Liu, Q. Song, and A. Jamalipour, "Turning the signal interference into benefits: Towards indoor self-powered visible light communication for IoT devices in industrial radio-hostile environments,' IEEE Access, vol. 7, pp. 24978-24989, Feb. 2019.

[27] I. Demirkol, D. Camps-Mur, J. Paradells, M. Combalia, W. Popoola, and H. Haas, "Powering the Internet of Things through light communication,' IEEE Commun. Mag., vol. 57, no. 6, pp. 107-113, Jun. 2019.

[28] Y. Almadani, M. Ijaz, S. Rajbhandari, B. Adebisi, and U. Raza, "Application of visible light communication in an industrial environment," in Proc. 11th Int. Symp. Commun. Syst., Netw. Digit. Signal Process., Jul. 2018, pp. 1-6.

[29] V. Jungnickel et al., "Optical wireless communication for backhaul and access," in Proc. Eur. Conf. Opt. Commun., Sep. 2015, pp. 1-3.

[30] P. W. Berenguer et al., "Optical wireless MIMO experiments in an industrial environment," IEEE J. Sel. Areas Commun., vol. 36, no. 1, pp. 185-193, Jan. 2018.

[31] P. W. Berenguer, J. Hilt, P. Hellwig, D. Schulz, J. K. Fischer, and V. Jungnickel, "Analog antenna diversity for reliable optical wireless communication systems," in Proc. Glob. LIFI Congr., Feb. 2018, pp. 1-5.

[32] P. W. Berenguer et al., "Real-time optical wireless mobile communication with high physical layer reliability," J. Lightw. Technol., vol. 37, no. 6, pp. 1638-1646, Mar. 2019.

[33] Unified high-speed wire-line based home networking transceivers - System architecture and physical layer specification, ITU-T Rec. G.9960 Corrigendum 1, Int. Telecommun. Union, Sep. 2019.

[34] P. W. Berenguer et al., "Real-time optical wireless communication: Fieldtrial in an industrial production environment," in Proc. Eur. Conf. Opt. Commun., Sep. 2018, pp. 1-3.

[35] V. Jungnickel et al., "LiFi for industrial wireless applications," in Opt. Fiber Commun. Conf., Mar. 2020.

[36] T. Fath and H. Haas, "Performance comparison of MIMO techniques for optical wireless communications in indoor environments," IEEE Trans. Commun., vol. 61, no. 2, pp. 733-742, Feb. 2013

[37] M. O. Damen, O. Narmanlioglu, and M. Uysal, "Comparative performance evaluation of MIMO visible light communication systems," in Proc. 24th Signal Process. Commun. Appl. Conf., May 2016, pp. 525-528.

[38] Q. H. Spencer, C. B. Peel, A. L. Swindlehurst, and M. Haardt, "An introduction to the multi-user MIMO downlink," IEEE Commun. Mag., vol. 42, no. 10 , pp. 60-67, Oct. 2004

[39] Y. Hong, J. Chen, Z. Wang, and C. Yu, "Performance of a precoding MIMO system for decentralized multiuser indoor visible light communications," IEEE Photon. J., vol. 5, no. 4, p. 7800211, Aug. 2013.

[40] Z. Yu, R. J. Baxley, and G. T. Zhou, "Multi-user MISO broadcasting for indoor visible light communication," in Proc. IEEE Int. Conf. Acoust., Speech Signal Process., May 2013, pp. 4849-4853.

[41] B. Li, J. Wang, R. Zhang, H. Shen, C. Zhao, and L. Hanzo, "Multiuser MISO transceiver design for indoor downlink visible light communication under per-LED optical power constraints," IEEE Photon. J., vol. 7, no. 4, pp. 1-15, Aug. 2015.

[42] M. K. Karakayali, G. J. Foschini, and R. A. Valenzuela, "Network coordination for spectrally efficient communications in cellular systems," IEEE Wireless Commun., vol. 13, no. 4, pp. 56-61, Aug. 2006.

[43] R. Irmer et al., "Coordinated multipoint: Concepts, performance, and field trial results," IEEE Commun. Mag., vol. 49, no. 2, pp. 102-111, Feb. 2011.

[44] V. Jungnickel et al., "The role of small cells, coordinated multipoint, and massive MIMO in 5G," IEEE Commun. Mag., vol. 52, no. 5, pp. 44-51, May 2014

[45] A. Checko et al., "Cloud RAN for mobile networks-A technology overview," IEEE Commun. Surv. Tut., vol. 17, no. 1, pp. 405-426, Jan.-Mar. 2015

[46] A. Maeder et al., "Towards a flexible functional split for cloud-RAN networks," in Proc. Eur. Conf. Netw. Commun., Jun. 2014, pp. 1-5.

[47] “ECPRI Specification V2.0," Accessed: Jul. 24, 2020. [Online]. Available: http://www.cpri.info/spec.html

[48] L. Zheng and D. N. C. Tse, "Diversity and multiplexing: A fundamental tradeoff in multiple-antenna channels," IEEE Trans. Inf. Theory, vol. 49, no. 5, pp. 1073-1096, May 2003.
[49] T. Le-Anh and M. D. Koster, "A review of design and control of automated guided vehicle systems," Eur. J. Oper. Res., vol. 171, no. 1, pp. 1-23, 2006.

[50] M. Aminikashani, W. Gu, and M. Kavehrad, "Indoor positioning with OFDM visible light communications," in Proc. 13th IEEE Annu. Consum. Commun. Netw. Conf., Jan. 2016, pp. 505-510.

[51] L. Yin, X. Wu, and H. Haas, "Indoor visible light positioning with angle diversity transmitter," in Proc. IEEE 82nd Veh. Technol. Conf., Sep. 2015 , pp. 1-5.

[52] J. Vongkulbhisal, B. Chantaramolee, Y. Zhao, and W. S. Mohammed, "A fingerprinting-based indoor localization system using intensity modulation of light emitting diodes," Microw. Opt. Technol. Lett., vol. 54, no. 5, pp. 1218-1227, 2012.

[53] A. Naz, H. M. Asif, T. Umer, and B.-S. Kim, "PDOA based indoor positioning using visible light communication," IEEE Access, vol. 6, pp. 7557-7564, Jan. 2018

[54] T.-H. Do, J. Hwang, and M. Yoo, "TDoA based indoor visible light positioning systems," in Proc. 5th Int. Conf. Ubiquitous Future Netw. Jul. 2013, pp. 456-458.

[55] S.-Y. Jung, S. Hann, and C.-S. Park, "TDOA-based optical wireless indoor localization using LED ceiling lamps," IEEE Trans. Consum. Electron. vol. 57, no. 4, pp. 1592-1597, Nov. 2011.

[56] G. F. Riley and T. R. Henderson, "The ns-3 network simulator," in Modeling and Tools for Network Simulation, Berlin, Heidelberg, Germany: Springer, 2010, pp. 15-34.

[57] A. Aldalbahi, M. Rahaim, A. Khreishah, M. Ayyash, and T. D. C. Little, "Visible light communication module: An open source extension to the ns3 network simulator with real system validation," IEEE Access, vol. 5, pp. 22144-22158, Oct. 2017.

[58] L. Yin, X. Wu, and H. Haas, "SDMA grouping in coordinated multi-poin VLC systems," in Proc. IEEE Summer Topicals Meeting Ser., Jul. 2015, pp. 169-170.

[59] C. Chen, Y. Yang, X. Deng, P. Du, and H. Yang, "Space division multiple access with distributed user grouping for multi-user MIMO-VLC systems," IEEE Open J. Commun. Soc., pp. 1-1, 2020.

[60] P. Djahani and J. M. Kahn, "Analysis of infrared wireless links employing multibeam transmitters and imaging diversity receivers," IEEE Trans. Commun., vol. 48, no. 12, pp. 2077-2088, Dec. 2000.

[61] S. Maravanchery et al., "LiFi experiments in a hospital," in Opt. Fiber Commun. Conf. Exhibit. (OFC), San Diego, CA, USA, 2020, pp. 1-3.

[62] S. M. Mana, S. M. Kouhini, P. Hellwig, J. Hilt, P. W. Berenguer, and V. Jungnickel, "Distributed MIMO experiments for LiFi in a conference room," presented at the 12th IEEE/IET Int. Symp. Commun. Syst., Netw. Digit. Signal Process., Porto Portugal, Jul. 2020.

[63] J. M. Kahn and J. R. Barry, "Wireless infrared communications," Proc. IEEE, vol. 85, no. 2, pp. 265-298, Feb. 1997.

[64] M. Hinrichs et al., "A physical layer for low power optical wireless communications," IEEE Trans. Green Commun. Netw., vol. 5, no. 1, pp. 4-17, Mar. 2021

[65] L. Grobe, V. Jungnickel, K.-D. Langer, M. Haardt, and M. Wolf, "On the impact of highpass filtering when using PAM-FDE for visible light communication," in Proc. IEEE Wireless Commun. Netw. Conf. Workshops, Apr. 2016, pp. 239-245.

[66] M. Wolf and M. Haardt, "On the DC balance of multi-level PAM VLC systems," in Proc. 21st Int. Conf. Transparent Opt. Netw., Jul. 2019, pp. 1-5.

[67] M. Hinrichs et al., "Pulsed modulation PHY for power efficient optical wireless communication," in Proc. 2019 IEEE Int. Conf. Commun., May 2019, pp. 1-7.

[68] M. Hinrichs et al., "Demonstration of optical wireless communications using the pulsed modulation PHY in IEEE 802.15.13," in Proc. 22nd Int. Conf. Transparent Opt. Netw. (ICTON), Bari, Italy, 2020, pp. $1-4$.

[69] M. Hinrichs, L. F. D. Rosal, C. Kottke, and V. Jungnickel, "Analog vs. next-generation digital fronthaul: How to minimize optical bandwidth utilization," in Proc. Int. Conf. Opt. Netw. Des. Model. (ONDM), May 2017, pp. 1-6.

[70] S. M. Kouhini et al., "Use of plastic optical fibers for distributed MIMO in Li-Fi systems," in Proc. Glob. LIFI Congr., 2019, pp. 1-5.

[71] S. M. Kouhini et al., "Performance of bidirectional LiFi over plastic optical fiber (POF)," presented at the 12th IEEE/IET Int. Symp. Commun. Syst., Netw. Digit. Signal Process., Porto, Portugal, Jul. 2020.

[72] A. Paraskevopoulos et al., "Design of a secure software-defined access network for flexible industry 4.0 manufacturing - The SESAM-project concept," in Proc. Glob. LIFI Congr., Jun. 2019, pp. 1-5. 
Kai Lennert Bober (Student Member, IEEE) received the M.Eng. degree in computer engineering from the Technical University of Berlin, Berlin, Germany, in 2018. In 2016, he joined the Department of Photonic Networks and Systems, Fraunhofer Institute for Telecommunications, Heinrich Hertz Institute, Berlin, Germany. He is currently working on optical wireless communications, including the development of prototypes and research towards realization aspects of distributed MIMO LiFi systems. He is also the Technical Editor of the IEEE P802.15.13 Task Group on multi-Gbit/s optical wireless communications.

Sreelal Maravanchery Mana received the M.Tech. degree in optical engineering from the Indian Institute of Space Science and Technology Trivandrum, Thiruvananthapuram, India, in 2017. He is currently working toward the Ph.D. degree in optical wireless communications. Since August 2018, he has been a Marie-Curie Research Fellow with the Department of Photonic Networks and Systems, Fraunhofer Institute for Telecommunications, Heinrich Hertz Institute, Berlin, Germany. His current research interests include the channel modelling simulation and experiments for optical wireless communication systems, MUMIMO for LiFi networks, and DCO-OFDM systems.

Malte Hinrichs (Member, IEEE) received the B.Eng. degree in electrical engineering from Duale Hochschule Baden-Württemberg Mannheim, Mannheim, Germany, in 2012, and the M.Sc. degree in electrical engineering from Technische Universität Berlin, Berlin, Germany, in 2017, where he is currently working toward the Dr.-Ing. degree. Since 2015, he has been with Fraunhofer HHI, Berlin, Germany, where he joined the Department on Photonics Networks and Systems, and since 2017, he has been with Technische Universität Berlin. His research interests include signal processing for energy efficient and robust OWC systems. He is a Member of the Verband der Elektrotechnik Elektronik Informationstechnik.

Sepideh Mohammadi Kouhini (Student Member, IEEE) received the B.S. degree in electronic engineering from Azad University, Tehran, Iran, and the M.S. degree in electronic engineering from ACECR University, Tehran, Iran. She has participated in several national and international projects in universities and research institutes, including APIC and VisIoN, Institute Telecommunication of Aveiro, Aveiro, Portugal, OSRAM Innovation Garching-Munich, Munich, Germany, and Fraunhofer Heinrich Hertz Institute (HHI), Berlin, Germany. She is currently a Ph.D. Researcher with HHI, working on VisIoN project as a Marie Sklodowska-Curie Innovative Training Network, a joint research training and doctoral programme with TU Berlin University. Her research interests include optical wireless communications and analog integrated design.

Christoph Kottke received the Dr.-Ing. (Ph.D.) degree from Technische Universität Berlin, Berlin, Germany, in 2019. Since 2010, he has been with the Fraunhofer Heinrich-Hertz-Institute, Berlin, Germany. His research interests include high-speed transmission systems for next-generation optical communication networks, and optical sensor systems for navigation and positioning.
Dominic Schulz received the M.Eng. degree in communications engineering from the Berlin University of Applied Sciences, Berlin, Germany, in 2012, and the Ph.D. degree from the Berlin Institute of Technology, Berlin, Germany, in 2019. In 2012, he joined the Department of Photonic Networks and Systems, Fraunhofer Institute for Telecommunications, Heinrich Hertz Institute, Berlin, Germany. He is currently working onoptical wireless communications. His current interests include the development of high data rate systems for wireless access and research toward the short-range outdoor backhaul links for small radio cells and wireless-to-the-home.

Christian Schmidt received the B.Sc. degree from Kiel University, Kiel, Germany, the M.Sc. degree from the Karlsruhe Institute of Technology, Karlsruhe, Germany, and the Dr. Ing. (Ph.D.) degree from Technische Universität Berlin, Berlin, Germany. Since 2015, he has been with the Fraunhofer Heinrich-HertzInstitute, Berlin, Germany. His current research interests include innovative optical wireless communication technologies, which include LiFi, and broadband signal generation for next generation optical communications networks.

Ronald Freund received the Dipl.-Ing. and Dr.-Ing. degree in electrical engineering from the Technical University of Ilmenau, Ilmenau, Germany, in 1993 and 2002, respectively, and the MBA degree from RWTH Aachen University, Aachen, Germany. In 1997, he co-founded VPI Systems Inc., where he was involved in as Chief Technology Officer and Consultant, responsible for the development of design software for the physical layer of photonic networks. Since 1995, he has been with Heinrich Hertz Institute, Berlin, Germany, where he is currently leading the Department of Photonic Networks and Systems. In 2017 he has been appointed as a Professor of photonic communication systems with the Technical University of Berlin, Berlin, Germany. He has authored or coauthored more than 150 scientific publications.

Volker Jungnickel (Member, IEEE) received the Doctoral degree from Humboldt University, Berlin, Germany, in 1995, and the Habilitation degree in physics and communications engineering from the Technical University of Berlin, Berlin, Germany, 2015, respectively. In 1997, he joined Fraunhofer HHI, Berlin, Germany, working on optical wireless communication, adaptive multiple antenna techniques in mobile networks, and fixed optical access infrastructures. $\mathrm{He}$ is with the Technical University of Berlin, Berlin, Germany, as Privatdozent teaching courses on advanced wireless communications and supervising master's and Ph.D. thesis. He is the Chair of IEEE P802.15.13 Task Group on multi-Gbit/s optical wireless communications and a Technical Editor of the IEEE P802.11bb Task Group on light communications. 